\title{
The Challenge of Tuberculosis Infection Related to Adalimumab: A Subtle and Rare Presentation
}

\author{
Juliana Lopes ${ }^{\mathrm{a}, \mathrm{e}}$, Ana Machado ${ }^{\mathrm{a}}$, Sara Martins ${ }^{\mathrm{a}}$, Sara Pires ${ }^{\mathrm{a}}$, Catarina Branco ${ }^{\mathrm{a}}$, \\ Joana Fragoso ${ }^{\mathrm{b}}$, Diogo Guerra ${ }^{\mathrm{b}}$, Patricia Campos ${ }^{\mathrm{c}}$, Mariana Brandao ${ }^{\mathrm{a}, \mathrm{d}}$, \\ Graziela Carvalheiras ${ }^{\mathrm{c}, \mathrm{d}}$, Teresa Mendonca ${ }^{\mathrm{a}, \mathrm{d}}$
}

\begin{abstract}
With the increasing use of biological therapies, there was a concomitant increase in concern and sensitivity for associated opportunistic infections. There has been growing evidence that anti-tumor necrosis factor (TNF) therapy increases the risk of opportunistic infection. Screening for latent tuberculosis (TB) prior to initiation of therapy has reduced the incidence of active TB, although it is not completely preventable. The possibility of active TB should always be considered in immunosuppressed patients under anti-TNF therapy. We report a case of muscular TB with psoas abscess in a patient under adalimumab. This case highlights the importance of the high suspicion threshold for Mycobacterium tuberculosis infection, in patients with subtle symptoms.
\end{abstract}

Keywords: Tuberculosis; Psoas muscle abscess; Anti-TNF; Adalimumab; Intestinal Behcet

\section{Introduction}

The use of anti-tumor necrosis factor (TNF) has dramatically improved the approach to immune-mediated diseases. However, there is a concomitant increase in concern and sensitivity for associated opportunistic infections [1]. These agents suppress activity of TNF, a proinflammatory cytokine crucial for human response to infection. In fact, several opportunistic infections have been described in association with therapy with anti-TNF $[2,3]$. The first randomized anti-TNF trial has described only one case of tuberculosis (TB) infection. Since then, there has been growing evidence that anti-TNF therapy increases the risk of TB infection, with a possible difference

Manuscript submitted December 3, 2017, accepted December 8, 2017

anternal Medicine Department, Centro Hospitalar do Porto, Porto, Portugal bInfectious Diseases Department, Centro Hospitalar do Porto, Porto, Portugal 'Intensive Care Department, Centro Hospitalar do Porto, Porto, Portugal ${ }^{\mathrm{d} C l i n i c}$ Immunology Unit, Centro Hospitalar do Porto, Porto, Portugal ${ }^{\mathrm{e} C}$ Corresponding Author: Juliana Lopes, Internal Medicine Department, Centro Hospitalar do Porto, Hospital de Santo Antonio, Largo Prof. Abel Salazar, 4099-001, Porto, Portugal. Email: mariajulopes@gmail.com

doi: https://doi.org/10.14740/jmc2979w between the three anti-TNF drugs used: infliximab and adalimumab with higher associated risk than etanercept $[4,5]$.

Screening for latent TB prior to initiation of therapy has reduced the incidence of active TB, although it is not completely preventable. There are cases of disease reactivation even after adequate treatment at the start of biological therapy. The possibility of active TB should always be considered in cases with suggestive clinical features or suspect imaging findings, even after chemoprophylaxis $[3,6]$. Portugal has an intermediate incidence of TB (22 cases per 100,000 inhabitants in 2016) compared to the rest of Europe; as a result, patients on anti-TNF therapy will have, therefore, a higher incidence of infection [7, 8]. Different from the presentation of the infection in immunocompetent patients, an infection by Mycobacterium tuberculosis associated with TNF blockade is more commonly as an extrapulmonary presentation. As described by Keane et al, of the 70 documented cases of TB infection with anti-TNF treatment, $57 \%$ were extrapulmonary and $25 \%$ disseminated [4].

We report a rare case of extrapulmonary TB in a patient using adalimumab.

\section{Case Report}

This was a 59-year-old woman, Caucasian, with a previous history of osteoarticular TB (Pott's disease) at the age of 7 years old. Since then, she had no history of infection or contact with other TB patients.

She was diagnosed as Behcet's disease with a 23-year-old history of outbreaks of oral ulcers. Since the age of 55 years, she started with severe gastrointestinal manifestations, irresponsive to corticosteroids and infliximab. She began adalimumab treatment 3 years ago with clinical improvement. Before anti-TNF therapy, she completed 9 months of isoniazid for latent tuberculosis.

The patient presented with high fever, asthenia and night sweats for 1 month, without other symptoms associated. Blood tests were innocent, with a slight protein $\mathrm{C}$ elevation. Blood and urine cultures were sterile. Pulmonary X-ray and abdominal ultrasound didn't reveal any findings. After 2 weeks of the first symptoms, she complained with left pain, without trauma associated. Computed tomography (CT) scan revealed a left psoas muscle mass (diameter $4-14 \mathrm{~cm}$ with calcifications) (Fig. 1). She was submitted to percutaneous drainage of the abscess and the microbiologic study revealed nucleic acid ampli- 


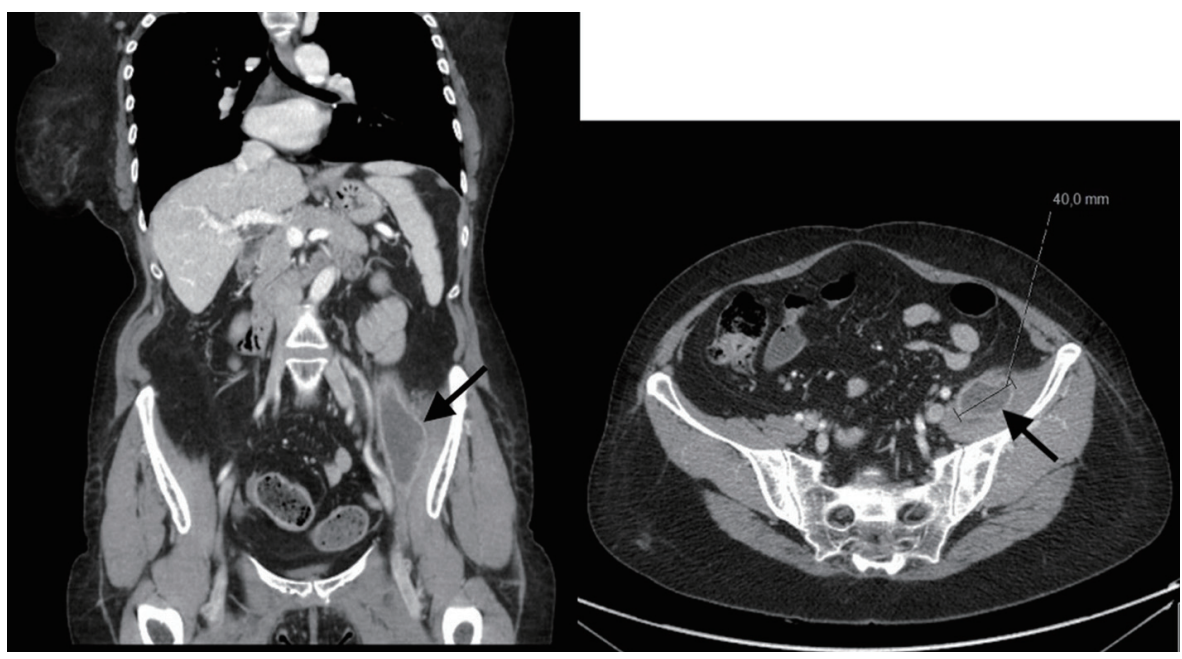

Figure 1. Abdominal CT scan showing left psoas muscle mass with the diameter $4-14 \mathrm{~cm}$. CT: computed tomography.

fication (NAA) test positive for Mycobacterium tuberculosis, which was also detected in cultures.

The patient began treatment with classic association of antibiotics that included isoniazid, rifampicin, pyrazinamide, and ethambutol for 9 months with complete resolution of the abscess (Fig. 2).

\section{Discussion}

The introduction of anti-TNF therapy drastically altered the approach to immune-mediated diseases. TNF plays a key role in host defence against Mycobacterium tuberculosis, mediating granuloma formation and homeostasis and consequent bacilli content in latent TB infection [9]. Consequently, a number of cases of TB have been documented in patients under antiTNF therapy such as etanercept, infliximab, and adalimumab [10]. This risk appears to be 3 to 4 times higher with infliximab and adalimumab when compared to etanercept [11]. It is believed that most cases are consecutive of latent TB reactivation and therefore, in countries with high or intermedium incidence, the screening is recommended before initiation of therapy and treated accordingly. Several studies have shown that chemoprophylaxis of latent TB before or during anti-TNF therapy reduces the risk of reactivation and consequently more severe forms of infection [9].

In clinical trials prior to the screening for TB, only 7 cases of active infection were reported in Europe. After the introduction of the screening in adalimumab clinical trials, there was an increase in the number of cases to 23 cases until 2005 and 59 cases until $2014[12,13]$.

In the present case, the active infection was documented despite screening and treatment of latent $\mathrm{TB}$, and is a rare extrapulmonary presentation.

The psoas abscess was first described in 1881[14] and remains a rare infection in developed countries. It can be termed as primary if the focus is unknown or by hematogenous spread or secondary if there is an abdominal or retroperitoneal infec-

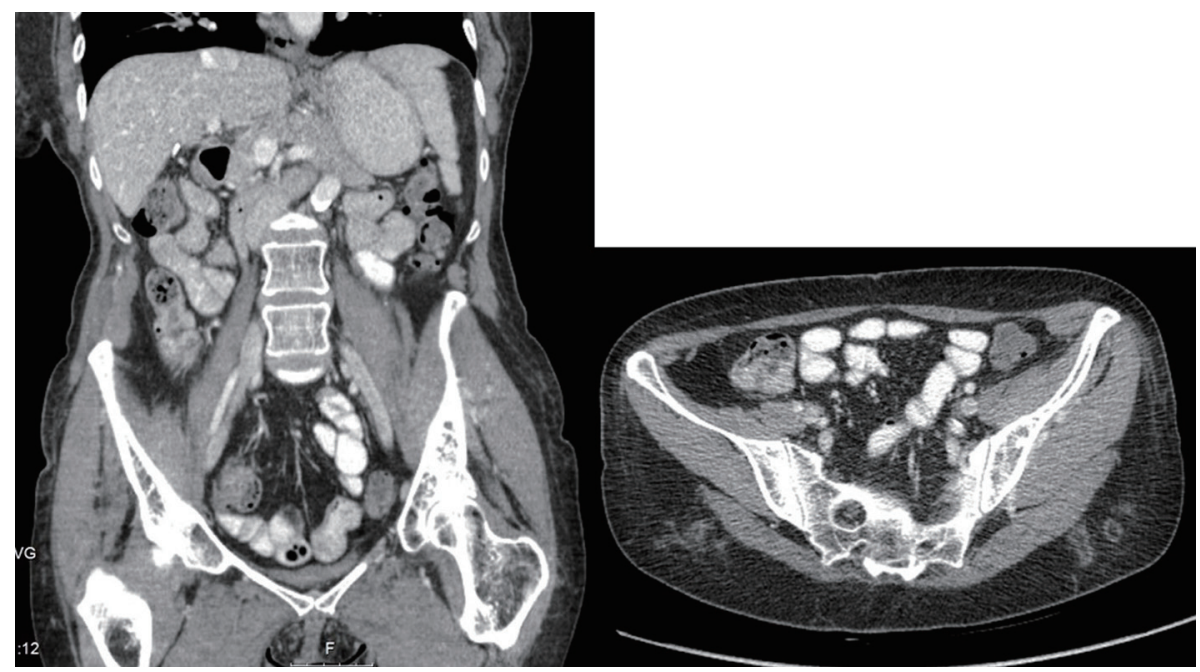

Figure 2. Abdominal CT scan with resolution of the abscess after 9 months of antibacillar therapy. 
tion. It is usually caused by Staphylococcus aureus, or other agents present in intestinal flora [15]. The immunosuppression is one of the major associated risk factors and for this reason, agents such as Mycobacterium tuberculosis should be considered.

Although several cases of extrapulmonary infection have been described [16-19], the authors only found one case of muscular TB in patients with adalimumab in literature [20].

This case highlights the importance of the high suspicion threshold for Mycobacterium tuberculosis infection. In immunocompromised patients, the clinical presentation is usually very subtle, extrapulmonary and rare forms are more frequent and may progress rapidly to more severe forms. Adequate early and therapeutic diagnosis will allow improving the longterm prognosis with resolution of the infection.

\section{Conflict of Interest}

There is no conflict of interest.

\section{Financial Support}

No external funds were used for this research study.

\section{References}

1. Sfriso P, Ghirardello A, Botsios C, Tonon M, Zen M, Bassi N, Bassetto F, et al. Infections and autoimmunity: the multifaceted relationship. J Leukoc Biol. 2010;87(3):385395.

2. Crum NF, Lederman ER, Wallace MR. Infections associated with tumor necrosis factor-alpha antagonists. Medicine (Baltimore). 2005;84(5):291-302.

3. Wallis RS, Broder MS, Wong JY, Hanson ME, Beenhouwer DO. Granulomatous infectious diseases associated with tumor necrosis factor antagonists. Clin Infect Dis. 2004;38(9):1261-1265.

4. Keane J, Gershon S, Wise RP, Mirabile-Levens E, Kasznica J, Schwieterman WD, Siegel JN, et al. Tuberculosis associated with infliximab, a tumor necrosis factor alphaneutralizing agent. N Engl J Med. 2001;345(15):10981104.

5. Mohan AK, Cote TR, Block JA, Manadan AM, Siegel JN, Braun MM. Tuberculosis following the use of etanercept, a tumor necrosis factor inhibitor. Clin Infect Dis. 2004;39(3):295-299.

6. Sichletidis L, Settas L, Spyratos D, Chloros D, Patakas D. Tuberculosis in patients receiving anti-TNF agents despite chemoprophylaxis. Int $\mathrm{J}$ Tuberc Lung Dis. 2006;10(10):1127-1132.

7. Rahier JF, Ben-Horin S, Chowers Y, Conlon C, De
Munter P, D’Haens G, Domenech E, et al. European evidence-based Consensus on the prevention, diagnosis and management of opportunistic infections in inflammatory bowel disease. J Crohns Colitis. 2009;3(2):47-91.

8. Global tuberculosis report 2017. Geneva: World Health Organization; 2017. Licence: CC BY-NCSA 3.0 IGO.

9. Gardam MA, Keystone EC, Menzies R, Manners S, Skamene E, Long R, Vinh DC. Anti-tumour necrosis factor agents and tuberculosis risk: mechanisms of action and clinical management. Lancet Infect Dis. 2003;3(3):148155 .

10. Keystone EC. Safety of biologic therapies - an update. J Rheumatol Suppl. 2005;74:8-12.

11. Dixon WG, Hyrich KL, Watson KD, Lunt M, Galloway J, Ustianowski A, Consortium BSRBRCC, et al. Drugspecific risk of tuberculosis in patients with rheumatoid arthritis treated with anti-TNF therapy: results from the British Society for Rheumatology Biologics Register (BSRBR). Ann Rheum Dis. 2010;69(3):522-528.

12. Schiff MH, Burmester GR, Kent JD, Pangan AL, Kupper H, Fitzpatrick SB, Donovan C. Safety analyses of adalimumab (HUMIRA) in global clinical trials and US postmarketing surveillance of patients with rheumatoid arthritis. Ann Rheum Dis. 2006;65(7):889-894.

13. Cantini F, Niccoli L, Goletti D. Adalimumab, etanercept, infliximab, and the risk of tuberculosis: data from clinical trials, national registries, and postmarketing surveillance. J Rheumatol Suppl. 2014;91:47-55.

14. Mynter H. Acute psoitis. Buffalo Med Surg J. 1881;21:202-210.

15. Ricci MA, Rose FB, Meyer KK. Pyogenic psoas abscess: worldwide variations in etiology. World J Surg. 1986;10(5):834-843.

16. Gutierrez-Macias A, Lizarralde-Palacios E, MartinezOdriozola P, Miguel-de la Villa F. Tuberculous peritonitis in a patient with rheumatoid arthritis treated with adalimumab. Clin Rheumatol. 2007;26(3):452-453.

17. Efde MN, Houtman PM, Spoorenberg JP, Jansen TL. Tonsillar tuberculosis in a rheumatoid arthritis patient receiving anti-TNFalpha (adalimumab) treatment. Neth J Med. 2005;63(3):112-114.

18. Lertxundi U, Mayo J, Garcia M, Ruiz B, Aguirre C. Tuberculoses miliar tras tratamiento con adalimumab. Farm Hosp. 2005;29(5):343-347.

19. Abreu C, Magro F, Santos-Antunes J, Pilao A, RodriguesPinto E, Bernardes J, Bernardo A, et al. Tuberculosis in anti-TNF-alpha treated patients remains a problem in countries with an intermediate incidence: analysis of 25 patients matched with a control population. J Crohns Colitis. 2013;7(10):e486-492.

20. Azevedo VF, Parchen C, Coelho SA, Lacerda DC, Hirth CG. Tuberculous myositis in a patient with ankylosing spondylitis treated with adalimumab. Rheumatol Int. 2009;29(11):1381-1384. 